\title{
Generic truth and mixed conjunctions: some alternatives*
}

AARon J. CotNoIR

Christine Tappolet [9] posed a problem for alethic pluralism: either deny the truth of conjunctions whose conjuncts are from distinct domains of inquiry, or posit a generic global truth property thus making other truth properties redundant. Douglas Edwards [2] has attempted to solve the problem by avoiding the horns of Tappolet's dilemma. After first noting an unappreciated consequence of Edwards' view regarding a proliferation of truth properties, I show that Edwards' proposal fails to avoid Tappolet's original dilemma. His response is not successful as it lets in a generic truth property through the 'back door'. I conclude by briefly offering a new solution to the problem, and an alternative diagnosis of Tappolet's dilemma.

\section{Tappolet's Dilemma}

The alethic pluralist contends that propositions from different domains can be true in different ways 1 Mixed conjunctions have conjuncts from different domains; consider for example, ' $1+1=2$ and murder is wrong'. A pressing question for the pluralist: if each conjunct is true in a distinct way, in what way is the conjunction true? Tappolet argues,

$[\mathrm{M}]$ ixed conjunctions need to be true in a further way. [...] But then each conjunct has to be true in the same way. This is what follows from the truism that a conjunction is true if and only if its conjuncts are true. Hence the question arises again why this further way of being true is not the only one we need. $([9,385)$

Edwards puts Tappolet's contention as a dilemma: either admit a generic truth property that can apply to all propositions, regardless of domain, or deny that

\footnotetext{
* forthcoming in Analysis 69.2, April 2008. (C)Aaron Cotnoir.
}

${ }^{1}$ E.g. Lynch [4], Sher 7], Wright [1]. 
mixed conjunctions can be true. It is prima facie plausible that mixed conjunctions can be true. Moreover, admitting a generic truth property would seemingly undermine alethic pluralism by making other truth properties redundant.

\section{Edwards' Solution}

Edwards' solution attempts to avoid both horns of the dilemma. He questions Tappolet's assumption that each conjunct must be true in the same way as the mixed conjunction itself.

The correspondence theory of truth endorses (1) and (2), identifying truth with the property of 'corresponding to a fact'.

(1) $p$ is true (corresponds to a fact).

(2) $q$ is true (corresponds to a fact).

But must the correspondence theorist also admit (3)?

(3) $p \& q$ is true (corresponds to a fact).

Edwards rightly notes that if the correspondence theorist does not admit conjunctive facts into her ontology, she is left in Tappolet's dilemma: either deny that conjunctions can be true or admit a non-correspondence notion of truth that applies to conjuncts as well as conjunctions. So, Tappolet's objection is a problem for pluralists only if it is a problem for other monistic theories of truth.

Edwards suggests that rather than accept the dilemma, the correspondence theorist can endorse (4).

(4) $p \& q$ is true by virtue of $p$ corresponding to a fact and $q$ corresponding to fact.

While the way in which $p \& q$ is true is derivative and entirely dependent on the truth of its conjuncts, the reverse direction of dependency does not hold. Edwards thinks a similar reply is available to the alethic pluralist. The pluralist can explain the truth of mixed conjunctions by (5).

(5) $p \& q$ is true by virtue of $p$ being true and $q$ being true.

Edwards admits that the conjunction must be true in some way distinct from the ways the conjuncts are true, but he denies that this third truth property needs to be had by each conjunct. Here lies a mistaken assumption in Tappolet's dilemma. 


\section{Generic Truth}

One is now left with a further question: just what is this distinct third way in which the conjunction is true? Since conjunctions must be true in some different way from atomics, one would like to know a bit more about the property. Edwards offers little explanation.

The further way that the conjunction is true is such that the truth of the conjunction is dependent on the truth of the individual conjuncts. $([2,148)$

In light of Edwards' solution for the correspondence theorist, it appears he must be thinking along the following lines.

(6) $p$ is true (is true 1 )

(7) $q$ is true (is true 2 )

(8) So, $p \& q$ is true by virtue of $p$ being true 1 and $q$ being true . $_{2}$

So the relevant truth property of this conjunction seems to be 'having a conjunct that is true ${ }_{1}$ and a conjunct that is true 2 '. But this property can only be had by conjunctions with conjuncts from domain 1 and domain 2. Clearly, we need some more general specification of the truth property had by all true mixed conjunctions if they are true in some distinct way.

An adequate property for true conjunctions would have to be something like true $_{c}$ : for any atomics $p$ and $q$ :

(9) $p \& q$ is true e $_{c} \quad$ iff $\quad p$ is in domain 1 and $p$ is true , $_{1}$ or $p$ is in domain 2 and $p$ is true 2 , $\ldots \quad$ or $p$ is in domain $n$ and $p$ is true ${ }_{n}$; and $\quad q$ is in domain 1 and $q$ is true 1 , or $q$ is in domain 2 and $q$ is true 2 , $\ldots \quad$ or $q$ is in domain $n$ and $q$ is true $_{n}$.

As desired, true ${ }_{c}$ is such that the truth of a conjunction is dependent on the truth of its conjuncts, without requiring that each conjunct itself have true . $^{\text {. }}$ However, there are two consequences of conjunctions having a property like true $_{c}$ that are worth highlighting. The first is that it leads to a proliferation of truth properties; the second is that it does not avoid a generic truth property. 
The first consequence is that Edwards' solution, and hence true ${ }_{c}$, is tailormade for conjunctions. If true ${ }_{c}$ is the truth property for conjunctions, what of other logical compounds like disjunctions, conditionals, etc.? If Edwards wishes to treat other truth-functional compounds in a parallel way, he will have an unappealing result: every distinct logical form will require a distinct truth property had by sentences of that form. Moreover, there are complications for iterated conjunctions. Consider a conjunction like $p \&(q \& r)$. What truth property will it have? Clearly, it cannot have true ${ }_{c}$, since the definition of true fails to include itself among the truth properties ${ }^{3}$ Must there be a new truth property for iterated conjunctions? Yes. Edwards admits in a footnote,

$[\mathrm{W}]$ hen one of the conjuncts is a conjunction, what makes that conjunct true (having each of its conjuncts being true) will be different from what makes a conjunct consisting of a singular proposition true (having the relevant truth property for its domain of discourse). ([2], 147 f. 8 )

Strictly speaking, only 'level 1' conjunctions (of atomics) will have true ${ }_{c}$. 'Level 2' conjunctions must have some other distinct truth property, 'level 3' conjunctions some further property, and so on. The result is a proliferation of truth properties. One might think that such a proliferation, however prima facie extreme, is part and parcel of truth pluralism, and that the given proliferation is thereby not a problem for Edwards' proposal 5 Without trying to decide the issue here, I shall only note two points about the first consequence of Edwards' proposal.

First, truth pluralists tend to think that the pluralism of truth arises from recognizably different metaphysical properties; none of them have suggested the infinite pluralism of truth 'properties' required by Edwards' solution. So, at the very least, Edwards' proposal increases the pluralism in truth pluralism in a surprising way and a simpler pluralism might be preferred. In $\S 4$, I offer one such pluralist solution that avoids proliferation of this kind. The second, related, point is simply that the proposal is against a 'sparse' conception of properties.

Whether, in the end, the first consequence is a problem or merely a surprise in Edwards' proposed solution is something I leave open. What is clearer is that the second consequence, to which I now turn, is a genuine problem with Edwards' proposal: his solution fails to get around Tappolet's dilemma. True ${ }_{c}$

\footnotetext{
${ }^{3}$ Hence, the relativization to atomic $p$ and $q$.

${ }^{5}$ Thanks to an anonymous referee for pointing this out.
} 
is itself a 'conjunctive property'; it has, intuitively speaking, two parts. The first part of the property - call it true $p_{p}$ - is as follows.

(10) $p$ is true e $_{p} \quad$ iff $\quad p$ is in domain 1 and $p$ is true ${ }_{1}$, or $p$ is in domain 2 and $p$ is true 2 , $\ldots \quad$ or $p$ is in domain $n$ and $p$ is true ${ }_{n}$.

Now, if the pluralist claims that true $e_{c}$ is the truth property for conjunctions, she will likewise hold that true $\mathrm{e}_{p}$ is a property of $p \mathrm{7}^{7}$ But notice that true $\mathrm{B}_{p}$ will be true of $p$ regardless of its domain. That is, true $_{p}$ is precisely the sort of generic truth property that constitutes the first horn of Tappolet's dilemma 8 If the pluralist admits that true ${ }_{c}$ is a genuine truth property for mixed conjunctions, she has let a generic truth property in 'through the back door'. And as far as I can see, any way of defining a truth property for mixed conjunctions along Edwards' lines will result in a generic truth property for all sentences.

\section{A New Solution and An Alternative Diagnosis}

There are very few solutions to Tappolet's dilemma proposed in the literature 9 In what follows, I provide a new solution to the problem that does not, as far as I can see, require a generic property of truth. And since it does not require the truth of conjunctions to be true in some distinct way, the new solution does not result in a proliferation of truth properties.

The solution trades on the fact that there are natural solutions to analogous problems with negations and mixed disjunctions. Mixed negations of atomics do not exist, and so a negation is true in the same way that its negand is false (or false in the same way the negand is true). That is, we allow $\sim p$ to have the truth property for the domain of $p$. For negations of compounds, the same solution is available: a negated compound is true in the same way(s) the compound is

\footnotetext{
${ }^{7}$ Moreover, if the considerations above are correct regarding Edwards' proliferation of truth properties, the pluralist will not be able to appeal to a sparse conception of properties in order to deny the existence of a property like true . $_{\text {. }}$

${ }^{8}$ Nikolaj Pedersen [6] has argued that alethic pluralists can avoid having a generic disjunctive property of truth only if they hold a sparse conception of properties. Clearly, in this case admitting true $e_{c}$ as a genuine property will require a less-than-sparse conception of properties. Indeed, all the same motivations for considering true ${ }_{c}$ a property will be motivation for taking true $_{p}$ to be a genuine property.

${ }^{9}$ Most of the literature is aimed at a related, yet different, problem of mixed inferences which is due to Tappolet [8]. See Beall [1], Lynch [3], and Pedersen [6]. The only published discussions of the mixed conjunction problem appear to be Williamson [10, Tappolet 9, Edwards [2] and Lynch [4]. Lynch's forthcoming book [5] also provides an extensive treatment.
} 
false (or false in the same way(s) the compound is true). The more difficult cases concern compounds. We might naturally suggest that a disjunction $p \vee q$ is true in the same way its true disjunct is. Supposing $p$ is in domain $m$ and $q$ in domain $n$, let the disjunction be true ${ }_{m}$ if $p$ is true ${ }_{m}$, and let it be true ${ }_{n}$ if $q$ is true ${ }_{n}$. If both disjuncts are true, let the disjunction be true in both ways. Likewise, if a disjunction is false, it is false in both ways the disjuncts are false.

These seem to be natural responses for negations and disjunctions. But De Morgan principles for propositional logic tell us that $p \& q$ is equivalent to $\sim(\sim p \vee \sim q)$. It is available to the pluralist simply to treat conjunction as a defined connective, and to suggest that mixed conjunctions are true in a parallel way to mixed disjunctions. So, $p \& q$ is true in the way that $\sim p \vee \sim q$ is false (by the truth conditions for negation). But $\sim p \vee \sim q$ must be false in the way that $\sim p$ is false and the way that $\sim q$ is false (by the falsity conditions for disjunction). We have it that $\sim p$ is false in the same way that $p$ is true, and $\sim q$ is false in the same way that $q$ is true (by the falsity conditions for negation). Therefore, $p \& q$ will have the truth property for $p$ and the truth property for $q$, but need not be true in some further way.

This solution seems promising as it does not generate a generic truth property, it requires no distinct truth property for compounds, and it treats all truth-functional connectives in a unified way 11 If, as Edwards appears to think, pluralists are required to do without a generic property of truth, there may be reason to explore this solution further.

I should note, however, that I do not endorse the above solution. This is because I see no reason to think that pluralists must avoid Tappolet's dilemma. In particular, while some pluralists reject the existence of a single generic truth property 12 I suggest that Tappolet is wrong to argue that a single generic property of truth would make other truth properties redundant.

Consider, for example, true $e_{p}$ above. An acceptance of true $e_{p}$ as a generic notion of truth would not make true $1, \ldots$, true $_{n}$ redundant or unnecessary. Since true $e_{p}$ is defined as a disjunction of true ${ }_{1}, \ldots$, true $_{n}$, its existence depends on these truth properties. On the contrary, it would seem that the existence of true $_{1}, \ldots$, true would make true $_{p}$ redundant, were it not for the problem of mixed conjunctions. In general, I suggest a pluralist can accept a generic truth property only if the generic property is defined by (or 'essentially' dependent

\footnotetext{
${ }^{11}$ Non-truth-functional compounds may well present further problems for pluralists, but discussion of this would be beyond the scope of this paper.

${ }^{12}$ For the most prominent example, see Wright's [11] denial of a generic truth property.
} 
on) the other truth properties.

Does accepting a generic truth property undermine the motivations for alethic pluralism? Pluralists often cite the varied and radical differences between propositions as their main motivation for positing multiple truth properties 14 But defining a generic property of truth by disjoining the many domain-relative truth properties hardly undermines this motivation. The disjunctive property is generic only because the domain-relative properties have already captured the differences between the domains. The charge is akin to claiming that there is no difference between apples and numbers since one can always define a property of being either an apple or a number.

Contrary to Edwards, his solution to the problem of mixed conjunctions does not avoid a generic truth property. What pluralists should learn from this, however, is that a generic truth property need not be avoided 15

\section{References}

[1] JC Beall. On mixed inferences and pluralism about truth predicates. The Philosophical Quarterly, 50:380-382, 2000.

[2] D. Edwards. How to solve the problem of mixed conjunctions. Analysis, 68.2:143-149, April 2008.

[3] M.P. Lynch. A functionalist theory of truth. In M.P. Lynch, editor, The Nature of Truth, pages 723-750. MIT Press, Cambridge, MA, 2001.

[4] M.P. Lynch. Truth and multiple realizability. Australasian Journal of Philosophy, 82(3):384-408, 2004.

[5] M.P. Lynch. Truth as One and Many: A Pluralist Manifesto. Oxford University Press, Oxford, forthcoming.

[6] N.J. Pedersen. What can the problem of mixed inferences teach us about pluralism? The Monist, 89(1):102-117, 2006.

[7] G. Sher. In search of a substantive theory of truth. The Journal of Philosophy, 101:5-36, 2004.

\footnotetext{
${ }^{14}$ See Lynch's scope problem 4, 5], or Sher's disunity challenge 7].

${ }^{15}$ Thanks to JC Beall, Colin Caret, Douglas Edwards, Michael Lynch, Nikolaj Pedersen, and Lionel Shapiro for valuable comments and discussion.
} 
[8] C. Tappolet. Mixed inferences: A problem for pluralism about truth predicates. Analysis, 57:209-210, 1997.

[9] C. Tappolet. Truth pluralism and many-valued logics: A reply to Beall. The Philosophical Quarterly, 50:382-385, 2000.

[10] T. Williamson. A critical study of truth and objectivity. International Journal of Philosophical Studies, 30:130-144, 1994.

[11] C. Wright. Truth and Objectivity. Harvard University Press, Cambridge, MA, 1992 . 